\title{
A Future Life Requires a Super Immune System
}

\author{
Majid Mohammed Mahmood
}

Department of Biology, College of Science, Mustansiriyah University. Baghdad, Iraq.

Corresponding author: majidmahmood93@yahoo.com

Received date: August 13, 2021; Accepted date: August 18, 2021 ; Published date: August 25, 2021

Citation: Majid MD. Mahmood (2021). A Future Life Requires a Super Immune System. J Clinical Research and Reports, 8(5); DOI:10.31579/26901919/197

Copyright: (c) 2021 Majid Mohammed Mahmood. This is an open access article distributed under the Creative Commons Attribution License, which permits unrestricted use, distribution, and reproduction in any medium, provided the original work is properly cited.
Abstract
Despite the capabilities of the immune system to qualify for saving the host through its adaptive and performance capabilities, many disease-causing organisms have adapted in ways that ensure their persistence. This necessitates developing immune system enhancements which may require fundamental modifications that aren't limited to routine procedures.
Key words: unpredictable mutations; treatment methods; immune system; disease-causing organisms; spread of pathogens; genetic manipulation

\section{Summary}

Despite the human use of prevention and treatment methods in their various forms. And despite the capabilities of the immune system to qualify for saving the host through its adaptive and performance capabilities, many disease-causing organisms have adapted in ways that ensure their persistence.

Some organisms adapt through excessive evasion of the immune system and disruption of some of its mechanisms, and some have developed abilities to invade and immerse in the host's organs...and so on. And it became not excluded that mutations would occur that lead to mutants/variants, which are beyond the range of the immune system's available capabilities, which is mainly burdened with an atmosphere saturated with pollutants and with the spread of pathogens in addition to the modern lifestyles accompanying them.

This necessitates developing immune system enhancements which may require fundamental modifications that aren't limited to routine procedures, (that may reach limits of genetic manipulation even) allowing the immune system to rise above the probable scenarios without relying on traditional immunization methods like vaccines, which may become impractical in the face of some of the strange and "unpredictable mutations".

This demands directing attention to studies that involve such endeavors to be pillars upon which subsequent steps are based. 